Revista de Investigación en Logopedia ISSN: $2174-5218$

http://dx.doi.org/10.5209/RLOG.59531

\title{
Voz cantada y Electromiografía de Superficie: Un estudio comparativo de la actividad muscular en cantantes formados y no formados
}

\author{
Clara Puig Herreros ${ }^{1}$; Vicent Rosell Clari ${ }^{2}$
}

Recibido: 11 de diciembre de 2017 / Aceptado: 23 de febrero de 2018

\begin{abstract}
Resumen. En los últimos años ha habido un incremento del uso de la Electromiografía de Superficie en logopedia, lo que se evidencia en el número creciente de publicaciones en este área en revistas científicas. Este instrumento permite presentar de forma objetiva y precisa la actividad mioeléctrica en tiempo real, es no invasiva y se percibe de fácil aplicación. El objetivo de este trabajo es comparar la actividad mioeléctrica de los músculos maseteros, suprahioideos y esternoicleidomastoideos de cantantes con y sin entrenamiento vocal reglado a través de la Electromiografía de Superficie durante la realización de un fonetograma. La muestra estuvo conformada por 12 sopranos con edades entre $19 \mathrm{y}$ 29 años, 6 con formación reglada en canto y 6 sin formación. Previo a la recogida de datos se calibró el sistema de análisis acústico a través de un calibrador y un dosímetro. Las muestras se recogieron en un gabinete insonorizado, previo consentimiento informado de los participantes. Se fijaron los electrodos en cada uno de los músculos, tanto derechos como izquierdos y se llevó a cabo el protocolo de registro de la actividad mioeléctrica. Se observaron diferencias estadísticamente significativas a nivel de los músculos esternocleidomastoideos, siendo mayor su actividad en los cantantes formados respecto a los no formados. No se obtuvieron diferencias significativas en los otros músculos estudiados. Puede concluirse que los cantantes con formación reglada presentan mayor actividad a nivel de músculos esternocleidomastoideos respecto a los no formados. Es necesario seguir investigando con muestras más amplias para llegar a resultados más concluyentes.
\end{abstract}

Palabras clave: Electromiografía de Superficie; voz; canto; músculos orofaciales.

\section{[en] Singing voice and Surface Electromyography: A comparative study of muscle activity in trained and non-trained singers}

Summary. In recent years, there has been an increase in the use of surface electromyography in speech therapy, which is evidenced in the growing number of publications in this area in scientific journals. This instrument can be objectively and accurately presented myoelectric activity in real time, non-invasive and perceived easy to apply. The aim of this work is to compare the myoelectric activity of the masseter, suprahyoid and sternomicleidomastoid muscles of singers with and without vocal training regulated through surface electromyography, during the performance of a phonetogram. The sample consisted of 12 sopranos with ages between 19 and 29 years, 6 with singing training and 6 without training. Prior to data collection, the acoustic analysis system was calibrated through a calibrator and a dosimeter. Samples were collected in a soundproof studio, with prior informed consent of the participants. The electrodes were fixed in each of the muscles, both right and left, and the protocol for recording myoelectric activity was carried out. Statistically significant differences were observed at the level

Clínica de Logopedia de la Fundació Lluís Alcanyís - Universitat de València

clara.puig@fundacions.uv.es

2 Departamento de Psicología Básica. Universitat de València

vicente.rosell@uv.es 
of the sternocleidomastoid muscles, with their activity being greater in the training singers compared to the non-training ones. No significant differences were observed in the other muscles studied. It has been found that singers with training have greater activity at the level of sternocleidomastoid muscles compared to the non-training ones. Further research is needed with larger samples to arrive at more conclusive results.

Keywords: Surface Electromyography; voice; singing; orofacial muscles.

Sumario: Introducción. Metodología. Resultados. Discusión. Limitaciones. Conclusiones. Referencias.

Cómo citar: Puig Herreros, C.; Rosell Clari, V. (2018). Voz cantada y Electromiografía de Superficie: Un estudio comparativo de la actividad muscular en cantantes formados y no formados. Revista de Investigación en Logopedia, 8(1), 77-89.

\section{Introducción}

La Electromiografía de Superficie (EMGS) viene siendo utilizada en el área de la fonoaudiología o la logopedia con la finalidad de conseguir datos más precisos y objetivos para ayudar a precisar el diagnóstico y planificar una intervención ajustada a cada caso en particular.

Según Balata, da Silva, de Moraes, Pernambuco y de Moraes (2013), existen dos métodos principales de exploración de la actividad eléctrica muscular. Por una parte, la electromiografía de inserción conocida también como Electromiografía (EMG), la cual se caracteriza por ser invasiva y utilizar electrodos a través de unas agujas finas insertadas en el músculo a estudiar. Por otro lado tenemos la EMGS, cuyos electrodos son fijados a la dermis para captar la actividad eléctrica muscular.

De esta manera, la EMGS es una herramienta segura, no invasiva y de fácil aplicación (Nakata, Jonsson y Hagner, 1993). Ha sido muy utilizada para conocer la fisiología de los músculos en tiempo real para el diagnóstico diferencial y el seguimiento de posibles trastornos musculares (Landulpho, Silva, Silva y Vitti, 2004).

Además de los electrodos de registro, es imprescindible la presencia de un electrodo de referencia, denominado electrodo de tierra, a fin de posibilitar la cancelación de interferencias, el cual se coloca alejado del punto muscular a estudiar.

La musculatura extrínseca laríngea también está involucrada en la fonación, especialmente los músculos suprahioideos $(\mathrm{SH})$ e infrahioideos $(\mathrm{IH})$, los cuales pueden ser evaluados por la EMGS (Balata et al., 2013).

En los últimos años se observa un incremento en el número de investigaciones que utilizan la EMGS para el estudio de la deglución, la masticación, la respiración, el canto, la voz normal y patológica, y la articulación del habla. Este aumento en el uso de la EMGS se debe a sus características, ya que es una técnica no invasiva, se percibe como de fácil aplicación y aporta información sobre la actividad muscular en tiempo real (Rosell-Clari, 2017).

Pettersen, Bjørkøy, Torp y Westgaard (2005) defienden la idea de que la presión subglótica es muy importante en la fonación y estudiaron la participación de los músculos esternocleidomastoideos (ECOM) en tareas de control del soplo y vocalización. Los resultados del estudio mostraron que los músculos ECOM y escalenos se activaban durante la inspiración y la fonación, observándose patrones de activi- 
dad correlacionados con la respiración. Se observó una mayor actividad electromiográfica en los músculos que contribuyen en la regulación de la presión subglótica (ECOM, escaleno y trapecio), influenciados por la demanda respiratoria, ya que el canto exige cerca del $100 \%$ de la capacidad vital frente al $50 \%$ del habla normal. Según estos autores, parece ser que la actividad del ECOM y el escaleno en canto clásico tienen una gran importancia funcional en el equilibrio de la presión subglótica. Pettersen y Westgaar (2005), también concluyen que los músculos ECOM y escalenos muestran patrones de actividad correlacionados durante la inspiración y la fonación en los cantantes clásicos.

Otros autores como Sapir y Larson (1993) estudiaron la actividad mioeléctrica durante el control del vibrato en maseteros, músculos perilaríngeos y periorales.

Balata, da Silva, Pernambuco, de Oliveira y de Moraes (2015) estudiaron los músculos laríngeos extrínsecos mediante EMGS para medir su actividad mioeléctrica durante la fonación. Estos autores manifiestan que existe una alta variabilidad en los registros electromiográficos intersujetos. Esta variabilidad puede dificultar la interpretación de la señal mioeléctrica y limitar las comparaciones entre otros sujetos debido a las diferencias a nivel fisiológico que subyacen a la evaluación de la señal eléctrica de los músculos a estudiar.

La EMGS se ha utilizado para evaluar los músculos laríngeos extrínsecos durante la masticación, la deglución, la voz y el habla, pero la discusión de los procedimientos para la normalización es controvertida y ha sido más discutida en estudios sobre las primeras dos funciones (Balata et al., 2015).

Khoddami, Talebian, Izadi y Ansari (2017) consideran que el uso de la EMGS es una herramienta que permite objetivar la tensión muscular en la disfonía. Estos mismos autores afirman que no es una herramienta fiable para distinguir sujetos con disfonía por tensión muscular (MDT) primaria de sujetos sanos.

Zhu et al. (2017) también estudiaron la actividad eléctrica de los músculos suprahioideos e infrahioideos en diferentes tareas de fonación. Según estos autores, la EMGS parece ser una herramienta potencial para evaluar la naturaleza dinámica de actividades musculares en patología de la voz. Estos autores centraron su estudio en la técnica HD EMGS (electromiografía de superficie HD con mapas topográficos), la cual fue utilizada para investigar la actividad muscular de los músculos laríngeos durante la fonación. Esta es una técnica novedosa para visualizar continuamente la actividad muscular global asociada a la fonación en la región anterior del cuello.

Toledo y Dalva (2001) describen que la musculatura extrínseca de la laringe tiene una función indirecta durante la fonación, siendo responsable de su anclaje en el cuello, tirando de la laringe y el hioides en todas direcciones. Dicha musculatura se divide en músculos $\mathrm{SH}$ e $\mathrm{IH}$, incidiremos más en los $\mathrm{SH}$ porque forman parte de este estudio.

Por otro lado, el músculo masetero se divide en dos fascículos: uno superficial y otro profundo. Éste último se inserta por arriba en los tres cuartos anteriores del borde inferior de la apófisis temporal del hueso cigomático. La función principal del masetero es elevar la mandíbula (Le Huche, Allali y Prado Marcilla, 2003) y contribuir en su proyección anterior y lateralización (Toledo y Dalva, 2001).

La activación aislada de la musculatura orofacial es difícil de adquirir, pero la actividad eléctrica muscular del masetero se puede registrar fácilmente porque es un músculo grande, fácil de palpar y ubicado de forma superficial (Stepp, 2012). 
Según autores como Brown, Hunt y Williams (1988), Shipp (1975) y Sundberg (1974), observaron que los cantantes no entrenados solían tener su laringe en una posición elevada mientras que los cantantes entrenados rara vez tenían su laringe posicionada por encima de su posición de reposo fisiológico.

Una de las hipótesis planteadas ha sido la existencia de una mayor actividad eléctrica muscular a nivel de músculos maseteros en los cantantes formados respecto a los no formados. En los estudios superiores de canto, sobretodo en el canto lírico, se pretende que los cantantes formados canten con una mayor apertura bucal para una mejor calidad de la voz (Bonet, 2004) y se les adiestra para ello. Para aumentar la apertura de la boca se recomienda la relajación de los músculos maseteros, pero durante el canto, la relajación mandibular se acompaña de un desplazamiento longitudinal voluntario hacia abajo, observándose un aumento de la actividad mioeléctrica en estos músculos.

Por otro lado, los músculos esternocleidomastoideos se insertan en el manubrio del esternón, en la clavícula y en la apófisis mastoides y participan en la realización de movimientos de cabeza (Toledo y Dalva, 2001). También es considerado un músculo accesorio en la respiración (Pettersen, 2005).

Aunque se ha documentado el aumento del uso de esta técnica en los últimos años, desde hace ya varias décadas, existen informes en la literatura científica que hablan sobre la dificultad metodológica para la recogida de datos electromiográficos, siendo el mayor dilema la falta de protocolos más específicos para llevar a cabo dicha aplicación (Türker, 1993).

Los estudios que se centran en investigar la función vocal son escasos y varían en función de los grupos musculares investigados, las técnicas utilizadas, si miden o no la musculatura cervical durante la fonación y el tamaño de la muestra, motivo por el cual es difícil comparar los resultados obtenidos en los diferentes estudios, no siendo posible estandarizar los procedimientos metodológicos utilizados en la evaluación (Balata, et al., 2013).

Aunque es cierto que el uso de esta técnica no está exenta de cierta dificultades, el uso de la EMGS puede dar una mejor información sobre el progreso del paciente, así como conocer la biomecánica muscular y ganar mayor objetividad en la investigación (Stepp, Heaton, Braden, Jetté, Stadelman-Cohen y Hillman, 2011; Balata, et al., 2013), frente a medidas táctiles o de observación, las cuales son subjetivas.

En este mismo sentido, Rosell-Clari (2017) después de valorar el uso de la EMGS como evaluación de la actividad eléctrica muscular y su uso rehabilitador como biofeedback concluye que mediante el uso de la EMGS se obtienen medidas fiables y se confirman las dificultades observadas en la exploración clínica miofuncional. Así mismos, el uso de la EMGS como biofeedback se muestra como una terapia muy útil para el autocontrol de la musculatura orofacial.

El objetivo principal de este trabajo es estudiar la actividad eléctrica muscular (medida en microvoltios) de los músculos maseteros, suprahioideos y esternocleidomastoideos de cantantes formados y no formados durante la realización del fonetograma a través de la EMGS.

Las hipótesis planteadas respecto a las medidas musculares fueron las siguientes: (a) Mayor actividad eléctrica muscular a nivel de músculos maseteros en los cantantes formados respecto a los no formados y (b) mayor actividad eléctrica muscular en suprahioideos y esternocleidomastoideos en cantantes no formados respecto a los formados. 
El estudio de la actividad eléctrica de estos músculos puede ayudar a la comprensión de la biomecánica de la fonación y aportar datos complementarios en investigaciones, evaluaciones e intervenciones en la práctica vocal (Stager, Bielamowicz, Regnell, Gupta, y Barkmeier, 2000; Rosell-Clari, 2017).

\section{Metodología}

Diseño. El diseño utilizado para este estudio fue cuasi experimental entresujeto. Cada paciente fue evaluado utilizando el mismo protocolo y posteriormente se analizaron los datos y se contrastaron a fin de valorar si existían diferencias significativas entre los sujetos.

Participantes. La muestra estaba conformada por dos grupos. Uno de ellos estaba compuesto por cantantes con formación reglada en canto lírico o cantantes profesionales y otro grupo compuesto por cantantes sin formación reglada o amateur, los cuales no han recibido ningún tipo de entrenamiento ni enseñanza vocal. Todos ellos participaron de manera totalmente voluntaria en este estudio y firmaron un consentimiento informado. Participaron un total de 12 mujeres que cantaban como sopranos con edades comprendidas entre 19 y 29 años, 6 con formación reglada y 6 sin formación en canto, a las que se les administró de forma individual y simultánea la técnica del fonetograma y la recogida de datos con la EMGS.

Todas ellas tenían buena salud, audición normal y libres de problemas vocales. También se registraron las características demográficas de cada una de ellas (edad, sexo, estatura y peso), imprescindibles para el uso de la EMGS.

En este estudio se siguieron las directrices éticas y legales vigentes en cuanto a investigación con seres humanos y protección de datos personales.

Materiales e intrumentos de medida. Se usó un micrófono Shure 849 para registrar la voz de los sujetos durante la recogida de las muestras de voz cantada. Éste estaba colocado sobre un pie de micrófono a la altura de la boca del sujeto, a unos $30 \mathrm{~cm}$ de distancia. El micrófono estaba conectado a una tarjeta de sonido (IMG Stage Line ${ }^{\circledR} M M X-24$ USB) con 10 canales y ésta a un ordenador fijo Lenovo con un sistema operativo Windows 7. Las muestras de voz fueron registradas y analizadas mediante el software informático de análisis acústico PRAAT (Boersma y Weenink, 2008, versión 2016).

Previamente a las grabaciones de voz, se utilizó un sonómetro (Q-100 Noise Dosimeter) y un calibrador acústico (Quest Technologies QC-10/QC-20) con una fuente de sonido de $1000 \mathrm{~Hz}$ a $114 \mathrm{~dB}$ para calibrar acústicamente la entrada de sonido al ordenador y asegurarnos de que no había distorsiones en los datos recogidos y posteriormente analizados.

Para recoger de manera objetiva los datos de la actividad eléctrica muscular se utilizó el electromiógrafo MioTool Face, de Miotec Suite 1.0. y un ordenador portátil Lenovo M30-70 con un sistema operativo Windows 8.1. Para recoger dicha actividad se utilizaron 13 electrodos (Meditrace) por cada sujeto y los resultados obtenidos se analizaron a través del software Miograph que incorpora el electromiógrafo. Para ayudar con el registro de la voz se utilizó un piano eléctrico (Casio CTK-720).

El análisis de los datos así recogidos se llevó a cabo mediante el programa informático IBM SPSS Statistic 22.0 (IBM, 2013). 
Procedimiento. Las muestras se recogieron en un gabinete que reúne unas condiciones acústicas adecuadas para el experimento, ya que tiene un nivel de insonorización procedente de fuentes externas de $60 \mathrm{~dB}$.

El procedimiento que se llevó a cabo para la recogida de muestras fue el siguiente: en primer lugar, el sujeto firmó el consentimiento informado que nos permitió seguir adelante con la recogida de muestras y luego, se le colocaron los electrodos en cada uno de los siguientes músculos: maseteros, suprahioideos y esternocleidomastoideos, tanto derecho como izquierdo siguiendo las recomendaciones de Balata, et al., (2013).

Se utilizaron seis canales de electromiografía (uno para cada músculo) y electrodos deshechables de material hipoalergénico para la captura de la señal eléctrica del músculo.

Gracias al software integrado en el electromiógrafo Miograph, se pudo analizar la actividad eléctrica muscular, permitiéndonos visualizar dicha actividad en tiempo real.

El protocolo que se siguió con la EMGS fue el siguiente:

En primer lugar, antes de adherir los electrodos, se limpiaron las regiones de los músculos maseteros, suprahioideos y esternocleidomastoideos con una gasa mojada en alcohol etílico hidratado de $70^{\circ}$. Con ello, se pretendió disminuir la impedancia de la piel para captar mejor la señal electromiográfica de los músculos seleccionados y así ofrecer una mayor condición de fijación de los electrodos (da Silva, 2013).

A continuación, se pasó a la colocación de los electrodos:

- Electrodo de referencia: cara interna del brazo izquierdo (cerca de la muñeca).

- Canal 1: Masetero izquierdo

- Canal 2: Masetero derecho

- Canal 3: Suprahioideo izquierdo

- Canal 4: Suprahioideo derecho

- Canal 5: Esternocleidomastoideo izquierdo

- Canal 6: Esternocleidomastoideo derecho

La actividad eléctrica se recogió a través del electromiógrafo conectado a un ordenador mediante un cable USB.

Una vez preparados, se pasó a registrar la actividad eléctrica muscular a través del siguiente protocolo:

1) 3 segundos de reposo y a continuación habla espontánea (nombre, apellidos, edad y fecha del día).

2) 3 segundos de reposo y a continuación cantar un pequeño fragmento (ad libitum), unos 60 segundos como máximo.

3) 3 segundos de reposo y a continuación una escala ascendente forte.

4) 3 segundos de reposo y a continuación una escala descendente forte.

5) 3 segundos de reposo y a continuación una escala ascendente piano.

6) 3 segundos de reposo y a continuación una escala descendente piano.

7) Para finalizar, 3 segundos de reposo y a continuación cantar otro pequeño fragmento (ad libitum), unos 60 segundos como máximo.

Aunque se registraron diferentes muestras vocales, en esta investigación tan solo se presentan los datos obtenidos en el fonetograma. 
Para proceder con el fonetograma se le pidió al sujeto que se pusiese de pie y emitiese una vocal /a/ delante del micrófono (a unos $30 \mathrm{~cm}$ de distancia). Se empezó por una nota media en la que el sujeto se encontraba cómodo, primero con la mayor intensidad posible y luego con la mínima que le fue factible. Primero fuimos ascendiendo nota a nota (do, mi, sol) en diferentes octavas hasta la frecuencia más alta que el sujeto pudo emitir y descendiendo luego hasta la más grave, independientemente de la calidad del timbre. Se le facilitaron todas las notas musicales con el piano.

Una vez registrada y analizada la voz, se realizó el fonetograma de forma manual, donde se colocó la máxima y la mínima intensidad en el eje de ordenadas y las diferentes frecuencias o alturas tonales en el eje de abscisas.

Se realizaron 12 fonetogramas, uno por cada cantante, donde se registró la emisión de un conjunto de frecuencias emitidas entre las notas Do2 $(131 \mathrm{~Hz})$ y Sol5 $(1.568 \mathrm{~Hz})$, dependiendo de la extensión tonal de cada sujeto.

Con la unión de ambos perfiles se obtuvo lo que se conoce como campo vocal, donde el límite izquierdo hacía referencia a la frecuencia más grave que la persona podía emitir y el límite derecho se correspondía con las frecuencias más agudas que podía hacer.

El análisis de la voz se realizó a través del software de análisis acústico PRAAT, estudiando el parámetro acústico de intensidad en decibelios (máxima y mínima) de todas las notas de las cuatro grabaciones del fonetograma (ascendente forte, descendente forte, ascendente piano y descendente piano).

El tiempo total del registro fue de unos 30 minutos aproximadamente por sujeto. Una vez finalizado, se guardaron todos los datos y se les retiraron los electrodos.

Cuando se obtuvieron todas las muestras de actividad eléctrica muscular y de voz se pasó al análisis individual de cada una de ellas.

El interés de este trabajo no es comparar si existen diferencias significativas entre cada una de las notas que producen los cantantes formados y no formados, sino comparar los resultados de forma global, siguiendo los objetivos e hipótesis de partida.

\section{Resultados}

El programa informático Miograph, que acompaña al electromiógrafo de superficie utilizado en esta investigación, permite el registro de la actividad eléctrica muscular de más de 400 muestras por segundo. Estos datos se pueden analizar directamente mediante el software Matlab, pero esta no fue la metodología utilizada porque el número de muestras obtenido era muy elevado. Se recogió toda la actividad microeléctrica, mezclándose los periodos de reposo con periodos de actividad eléctrica no deseada (por ejemplo al tragar saliva) y la actividad eléctrica muscular objeto de estudio, cuando se realizaron las distintas notas del fonetograma. Para superar esta dificultad, se seleccionaron los periodos temporales de actividad microeléctrica de interés, de cada uno de los músculos seleccionados y se registraron los parámetros que proporciona directamente el electromiógrafo de superficie: Máxima $(\mu \mathrm{V})$, media $(\mu \mathrm{V})$, mínima $(\mu \mathrm{V})$ y desviación típica $(\mu \mathrm{V})$.

De este modo se obtuvieron los resultados descriptivos que se presentan en la Tabla 1. En esta tabla se recogen las medias aritméticas de la actividad eléctrica muscular correspondiente a cada uno de los músculos objeto de estudio, de los can- 
tantes formados y no formados, mientras producen las notas correspondientes al fonetograma fuerte y al fonetograma piano. En la Tabla 1 se observa mayor actividad microeléctrica en los maseteros derecho e izquierdo durante la realización del fonetograma fuerte en los cantantes formados respecto a los no formados.

Tabla 1. Estadísticos descriptivos

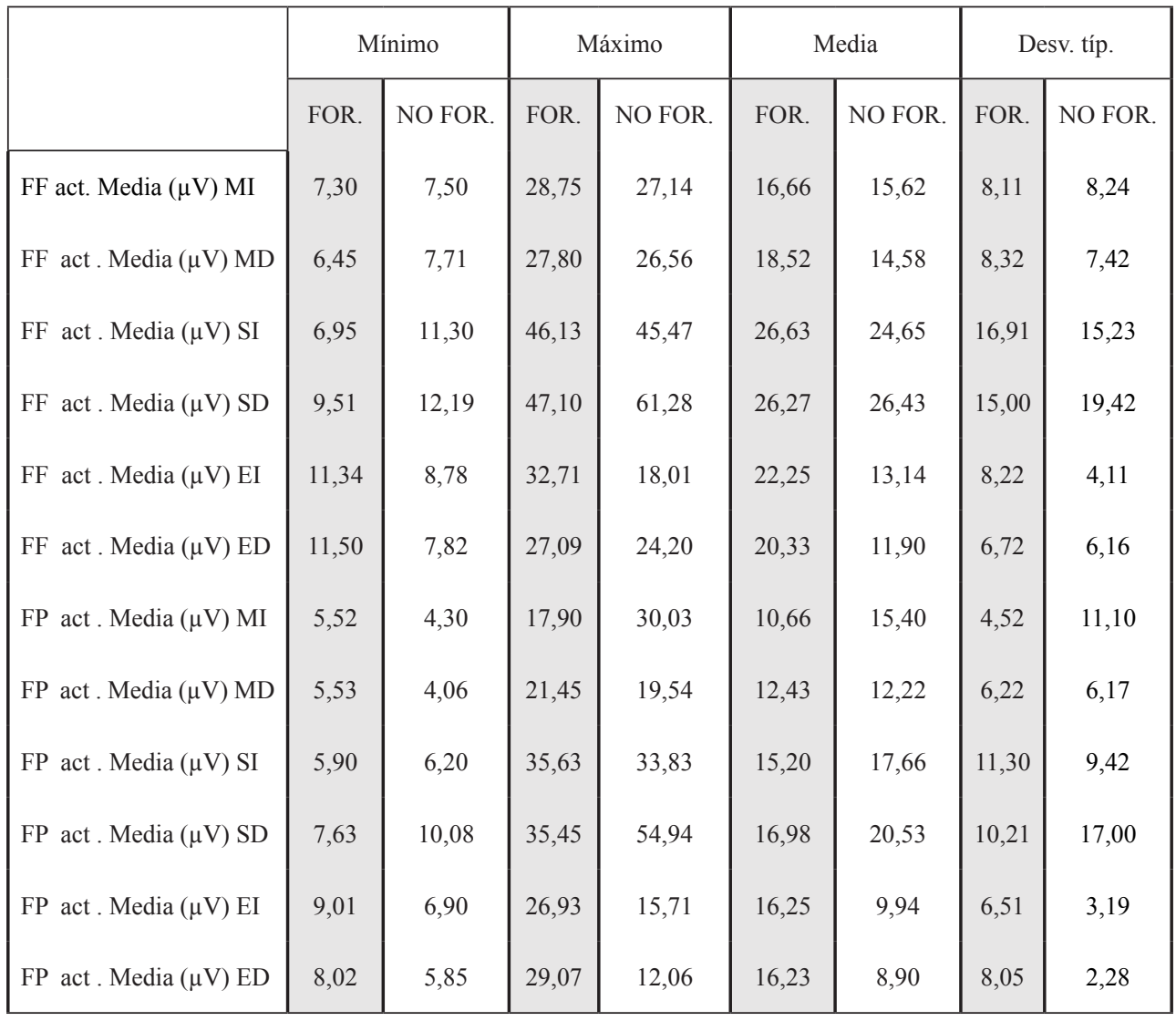

FOR: cantantes formados; NO FOR: cantantes no formados; FF: fonetograma fuerte; FP: fonetograma piano; act. Media: activación muscular media; MI: masetero izquierdo; MD: masetero derecho; SI: suprahioideo izquierdo; SD: suprahioideo derecho; EI: esternocleidomastoideo izquierdo; ED: esternocleidomastoideo derecho; $\boldsymbol{\mu V}$ : microvoltios.

Previamente a contrastar la actividad microeléctrica de las diferentes variables en función de la formación se valoró si las variables dependientes seguían la distribución normal a través de la prueba no paramétrica de Kolmogorov-Smirnov. Los resultados que se obtuvieron se presentan en la Tabla 2 y confirman que todas las variables siguen la distribución normal, es por ello que para valorar si existían diferencias significativas en función de la formación de las cantantes, se realizó una ANOVA de un factor tomando como variable independiente la variable «formación» y todas las demás como variables dependientes. 
Además, en la Tabla 2 se muestran diferencias estadísticamente significativas en los resultados del fonetograma fuerte en la actividad eléctrica muscular media de los músculos esternocleidomastoideos izquierdo $\left(\mathrm{F}_{(1,11)}=5.895 ; \mathrm{p}=0.036\right)$ y derecho $\left(F_{(1,11)}=5.122 ; p=0.047\right)$, siendo mayores los resultados en los cantantes formados.

Tabla 2 Anova de un factor

\begin{tabular}{|l|c|c|c|c|}
\hline \multirow{2}{*}{$\begin{array}{l}\text { MÚSCULOS } \\
\text { (actividad media en } \mu \mathrm{V})\end{array}$} & \multicolumn{2}{|c|}{ FONETOGRAMA FUERTE } & \multicolumn{2}{|c|}{$\begin{array}{r}\text { FONETOGRAMA } \\
\text { PIANO }\end{array}$} \\
\cline { 2 - 5 } & $\mathrm{F}(1,11)$ & $\mathrm{p}$ & $\mathrm{F}(1,11)$ & $\mathrm{p}$ \\
\hline Masetero izquierdo & 0,048 & 0,831 & 0,920 & 0,360 \\
Masetero derecho & 0,750 & 0,407 & 0,003 & 0,955 \\
Suprahioideo izquierdo & 0,046 & 0,835 & 0,167 & 0,692 \\
Suprahioideo derecho & 0,000 & 0,988 & 0,193 & 0,670 \\
$\begin{array}{l}\text { Esternocleidomastoideo } \\
\text { izquierdo }\end{array}$ & 5,895 & $\mathbf{0 , 0 3 6}$ & 4,549 & 0,059 \\
$\begin{array}{l}\text { Esternocleidomastoideo } \\
\text { derecho }\end{array}$ & 5,122 & $\mathbf{0 , 0 4 7}$ & 4,598 & 0,058 \\
\hline
\end{tabular}

Actividad eléctrica muscular media durante la realización de los fonetogramas fuerte y piano.

Respecto a la hipótesis (b), aunque en algunos músculos se observa que los cantantes no entrenados presentan mayor actividad microeléctrica que los entrenados, estos resultados no son estadísticamente significativos.

\section{Discusión}

Aunque las diferencias en la actividad eléctrica muscular de los maseteros derecho e izquierdo no son estadísticamente significativas, esta variabilidad se podría explicar de la siguiente manera: la acción principal conjunta de los músculos maseteros es la elevación de la mandíbula, permitiendo así el cierre de la boca. Cuando estos músculos se relajan, la mandíbula desciende y por tanto la boca se abre por acción de la gravedad. Como la boca es el principal resonador de la voz, su mayor o menor apertura será de gran importancia para la intensidad y el timbre. En el canto (al menos en el canto lírico), es necesario abrir la boca para crear una cavidad de resonancia adecuada a los tonos producidos por las cuerdas vocales (Torres, 2004).

La musculatura maseterina deberá estar relajada y de este modo se abrirá la boca sin producir tensiones (Bonet, 2004). Sin embargo, como ya se ha comentado, los cantantes formados, conocedores de la importancia de la apertura bucal durante el 
canto, realizan una apertura voluntaria en la que los músculos maseteros no están totalmente relajados, sino que se produce una extensión mandibular antagónica a la contracción mandibular.

Debemos tener en cuenta que durante la apertura oral que se utiliza en el canto lírico, el músculo masetero no está en reposo, sino que está elongado, dando lugar a una actividad eléctrica muscular mayor con respecto a aquellas personas que cantan con la boca más cerrada (menor actividad microeléctrica). La intensidad o volumen del sonido será directamente proporcional al área de apertura mandibular (Bonet, 2004).

Como ya se dijo anteriormente, los resultados estadísticamente no significativos pueden ser debidos a otras variables como el pequeño tamaño de la muestra, diferencias antropométricas como la altura, el peso, el tamaño y longitud de los músculos, que pueden interferir en la realización de mayor o menor actividad eléctrica muscular al cantar (Balata, da Silva, Pernambuco, de Oliveira y de Moraes, 2015).

El uso de la EMGS nos permite conocer con mayor profundidad la fisiología de los músculos, tanto en reposo como en actividad (como es en este caso el canto), en tiempo real y ello permite profundizar en el diagnóstico diferencial y en el seguimiento de posibles trastornos musculares (Landulpho, Silva, Silva y Vitti, 2004). También puede ayudar en la comprensión de la biomecánica de la fonación y aportar datos complementarios en la evaluación, diagnóstico e intervención (Stager, Bielamowicz, Regnell, Gupta, y Barkmeier, 2000; Rosell-Clari, 2017).

\section{Limitaciones}

Hay muy poca investigación sobre este tema y es difícil comparar resultados, ya que varían los grupos musculares analizados, el tamaño de la muestra, el método de estandarización, etc. El hecho de contar con una muestra pequeña de sujetos no permite generalizar los resultados $(\mathrm{N}=12)$.

Al igual que cualquier otro método, la aplicabilidad de la EMGS puede tener algunas limitaciones, como el espesor de la piel, la motivación del paciente para llevar a cabo el examen y otras variables no controladas.

\section{Conclusiones}

El objetivo de este estudio es comparar cantantes con formación reglada y sin formación reglada a nivel de actividad eléctrica muscular durante la ejecución de un fonetograma, medida a través de la EMGS, en los músculos maseteros, suprahioideos y esternocleidomastoideos.

Con todos los datos obtenidos se observa que se cumplen las hipótesis planteadas, excepto la hipótesis sobre la realización de mayor actividad microeléctrica en cantantes no formados respecto a los formados.

También hay datos que van en el mismo sentido, como la hipótesis (a), referente a la actividad eléctrica muscular realizada, en la que los cantantes formados presentan un mayor desplazamiento a nivel de maseteros respecto al otro grupo. Sin embargo, 
no se confirma la hipótesis (b), al menos para la mayoría de los músculos en los cantantes no entrenados.

Existen diferencias significativas en los resultados del fonetograma fuerte a nivel de actividad eléctrica muscular media de los músculos esternocleidomastoideos (tanto derecho como izquierdo), siendo mayor la actividad en los cantantes formados. Según Pettersen (2005), el músculo esternocleidomastoideo es considerado como apoyo del mecanismo fonatorio debido a la relación que presenta con la estructura de la laringe y del esternón. Hoit y Hixon (1986), citados por Pettersen (2005), indican que la alta actividad de estos músculos es una de las características de fatiga y tensión entre los cantantes. En algunas técnicas de canto, la elevación del esternón es relativamente alta (Manifold y Murdoch, 1993).

En logopedia, la EMGS se considera una ayuda importante en la comprensión de los patrones de actividad eléctrica de los músculos faciales y masticatorios, lo que contribuye a un diagnóstico e intervención más objetivo y eficaz (Ferla, Silva y Corrêa, 2008; Rosell-Clari, 2017), pues a diferencia de la palpación o la observación (técnicas más subjetivas), la EMGS permite complementar de forma objetiva los datos de diagnóstico, tratamiento, pronóstico e intervención logopédica.

Como el uso de esta herramienta todavía es relativamente nuevo en el campo de la logopedia, es razonable que actualmente haya pocos estudios en los que se utilice. La gran mayoría de los artículos donde se utiliza la EMGS en logopedia están relacionados con las áreas de motricidad orofacial. El área de la voz está menos estudiada en este aspecto, motivo por el cual requiere ser explorada e investigada más a fondo.

El aumento de su uso, no sólo en la literatura sino también en la práctica clínica, hace que sea de interés investigar el estado actual del conocimiento sobre esta técnica y su utilización en los estudios relacionados con la voz (Balata et al., 2013). Estos autores observaron que desde el año 2004 las publicaciones en esta área tuvieron una frecuencia anual, lo cual demuestra un creciente interés en la comunidad científica para contribuir en la objetividad de los datos y para comprender mejor el comportamiento de los músculos implicados en la fonación a través de la utilización del EMGS.

Muchos estudios remarcan la necesidad de establecer una normalización de los datos obtenidos de la señal electromiográfica, sobre todo para poder reproducir mejor los estudios.

Para finalizar, es importante remarcar que son necesarias un número mayor de investigaciones que estudien y utilicen la voz cantada junto con la EMGS para llegar a resultados más concluyentes.

\section{Referencias}

Balata, P. M. M., da Silva, H. J., Pernambuco, L. A., Amorim, G. O., Braga, R. S. M., Fernandes da Silva, E. G., de Lima, L. M., y Moraes, S. R. A. (2015). Electrical activity of extrinsic laryngeal muscles in subjects with and without dysphonia. Journal of Voice, 29(1), 129.e9 -129.e17. doi:10.1016/j.jvoice.2014.03.012

Balata, P. M. M, da Silva, H. J., Rocha de Moraes, K. J., Pernambuco, L. A., y de Moraes, S. R. A. (2013). Use of Surface electromyography in phonation studies: an integrative 
review. International Archives of Otorhinolaryngology, 17(3), 329-339. doi: 10.7162/ S1809-977720130003000014

Boersma, P., y Weenink, D. (2008). PRAAT (versión 2016) [Software de computación]. Institute of Phonetic Sciences: University of Amsterdam.

Bonet, M. (2004). Fisiología del canto. En Rumbau, J. (2004). La medicina del canto. Recuperado de http://www.medicinadelcant.com/

Brown, J. W. S., Morris, R. J., Hicks, D. M., y Howell, E. (1993). Phonational profiles of female professional singers and nonsingers. Journal of Voice, 7(3), 219-226. doi: 10.1016/ S0892-1997(05)80330-3

Brown, W. S., Hunt, E., y Williams, W. N. (1988). Physiological differences between the trained and untrained speaking and singing voice. Journal of Voice, 2(2), 102-110. doi: 10.1016/S0892-1997(88)80065-1

Ferla, A., da Silva, A. M. T., y Corrêa, E. C. R. (2008). Atividade eletromiográfica dos músculos temporal anterior e masseter em crianças respiradoras bucais e em respiradoras nasais. Revista Brasileira de Otorrinolaringologia, 74(4), 588-595.

Hoit, J. D., y Hixon, T. J. (1986). Body Type and Speech Breathing. Journal of Speech, Language, and Hearing Research, 29, 313-324. doi:10.1044/jshr.2903.313

IBM SPSS Statistics 22.0 (2013). [Software de computación]. Armonk, NY: IBM Corp.

Justino da Silva, H. (2013). Protocolos de electromiografia de superficie em fonoaudiologia. São Paulo: Pró-Fono departamento Editorial.

Khoddami, S. M., Talebian, S., Izadi, F., y Ansari, N. N. (2017). Validity and Reliability of Surface Electromyography in the Assessment of Primary Muscle Tension Dysphonia. Journal of Voice, 31(3), 386-e9. doi: 10.1016/j.jvoice.2016.09.010

Landulpho, A. B., Silva, W. A. B. E., y Silva, F. A. E., Vitti, M. (2004). Electromyographic evaluation of masseter and anterior temporalis muscles in patients with temporomandibular disorders following interocclusal appliance treatment. Journal of Oral Rehabilitation, 31(2), 95-98. doi: 10.1046/j.0305-182X.2003.01204.X

Le Huche, F., Allali, A., y Prado Marcilla, J. M. (2003). La voz: Anatomía y Fisiología de los Órganos de la Voz y del Habla. Volumen 1. Barcelona: Masson.

Manifold, J. A. Y., y Murdoch, B. E. (1993). Speech Breathing in Young Adults: Effect of Body Type. Journal of Speech, Language, and Hearing Research, 36, 657-671. doi:10.1044/jshr.3604.657

Nakata, M., Jonsson, B., y Hagner, I.M. (1993). Trapezius muscle pressure pain threshold and strain in the neck and shoulder regions during repetitive light work. Scandinavian Journal of Rehabilitation Medicine, 25(3), 131-137.

Pernambuco, L., da Cunha, R. A., Lins, O., Leão, J. C., y da Silva, J. H. (2010). A electromiografía de superficie nos periódicos nacionais em fonoaudiologia. Revista CEFAC, 4(12). doi: 10.1590/S1516-18462010005000082

Pettersen, V., Bjørkøy, K., Torp, H., y Westgaard, R. H. (2005). Neck and shoulder muscle activity and thorax movement in singing and speaking tasks with variation in vocal loudness and pitch. Journal of Voice, 19(4), 623-634. doi: 10.1016/j.jvoice.2004.08.007

Pettersen, V. (2005). Muscular patterns and activation levels of auxiliary breathing muscles and thorax movement in classical singing. Folia Phoniatrica Et Logopaedica, 57(5-6), 255-277. doi:10.1159/000087079

Pettersen, V., y Westgaard, R. H. (2005). The activity patterns of neck muscles in professional classical singing. Journal of Voice, 19(2), 238-251. doi:10.1016/j.jvoice.2004.02.006

Rosell-Clari, V. (2017). Alteraciones orofaciales y electromiografía de superficie en trastornos del neurodesarrollo. Revista de Neurología, 64(1), 84-88. 
Sapir, S., y Larson, K. K. (1993). Supralaryngeal muscle activity during sustained vibrato in four sopranos: surface EMG findings. Journal of Voice, 7(3), 213-218. doi: 10.1016/ S0892-1997(05)80329-7

Shipp, T. (1975). Vocal frequency and vertical larynx positioning by singers and nonsingers. The Journal of the Acoustical Society of America, 58(5), 1104-1106. doi:10.1121/1.380776

Stager, S. V., Bielamowicz, S. A., Regnell, J. R., Gupta, A., y Barkmeier, J. M. (2000). Supraglottic Activity: Evidence of Vocal Hyperfunction or Laryngeal Articulation? Journal of Speech, Language, and Hearing Research, 43, 229-238. doi:10.1044/jslhr.4301.229

Stepp, C. E. (2012). Surface electromyography for speech and swallowing systems: measurement, analysis, and interpretation. Journal of Speech, Language, and Hearing Research, 55(4), 1232-1246. doi:10.1044/1092-4388(2011/11-0214)

Stepp, C. E., Heaton, J. T., Braden, M. N., Jette, M. E., Stadelman-Cohen, T. K., y Hillman, R. E. (2011). Comparison of neck tension palpation rating systems with surface electromyographic and acoustic measures in vocal hyperfunction. Journal of Voice, 25, 67-75. doi: 10.1016/j.jvoice.2009.08.001

Stepp, C. E., Heaton, J. T., Jette, M. E., Burns, J. A., y Hillman, R. E. (2010). Neck surface electromyography as a measure of vocal hyperfunction before and after injection laryngoplasty. Annals of Otology, Rhinology and Laryngology, 119, 594-601.

Sundberg, J. (1974). Articulatory interpretation of the 'singing formant'. The Journal of the Acoustical Society of America, 55(4), 838-844. doi:10.1121/1.1914609

Toledo González, N. Z., y Dalva Lopes, N. (2001). Logopedia y ortopedia maxilar en la rehabilitación orofacial: tratamiento precoz y preventivo. Terapia Miofuncional. México: Actualidades Médico Odontológicas Latinoamérica.

Torres, B. (2004). Anatomía funcional de la voz. En Rumbau, J. (2004). La medicina del canto. Recuperado de http://www.medicinadelcant.com/

Türker, K. S. (1993). Electromyography: Some Methodological Problems and Issues. Journal of the American Physical Therapy Association, 73, 698-710. doi: 10.1093/ptj/73.10.698

Zhu, M., Liang, F., Samuel, O. W., Chen, S., Yang, W., Lu, L., Zou, H., Li, P., y Li, G. (2017, July). A pilot study on the evaluation of normal phonating function based on high-density sEMG topographic maps. In Engineering in Medicine and Biology Society (EMBC), 2017 39th Annual International Conference of the IEEE (pp. 1030-1033). IEEE. doi: 10.1109/ EMBC.2017.8037002 